\title{
Vier Briefe an Filippo und Rudolfino Venuti.
}

Mitgeteilt von RICH. ENGELMANN.

\section{Briefe des Baron Phil. v. Stosch.}

Zu den Briefen des Philipp v. Stosch, die im Archiv für Kulturgeschichte (Bd. 6, H. 3, S. 326 ff.) veröffentlicht sind, mögen hier einige Nachtrāge folgen. Es ist mir durch die Freundlichkeit der Marquise Teresa Venuti (die in einem Buche "I Venuti“, Roma 1889, die sāmtlichen Glieder der Familie Venuti ausführlicher behandelt hat) und des Cav. Girolamo Mancini in Cortona, des Prāsidenten der noch heute bestehenden Accademia Etrusca, gelungen, noch zwei (im folgenden als No. 1,2 abgedruckte) Briefe des Baron v. Stosch, die an Filippo Venuti gerichtet waren, zu erhalten. Der Einzug des Großherzogs Franz II. in Florenz, der im ersten jetzt veröffentlichten Briefe genauer mit allen Einzelheiten geschildert wird, ist von Marcus Tuscher in einem großen Kupferstich, der in London im Jahre 1743 veröffentlicht ist, genau geschildert worden.

Die zum besseren Verständnis nötigen Zusätze (Stosch schreibt das Französische so wie er spricht, ohne jede Rücksicht auf die übliche Orthographie) sind in eckige Klammern eingeschlossen, so daß die runden Klammern für die eigenen $\mathrm{Zu}$ sätze des Briefschreibers bleiben.

Weiter mag noch ein an Rudolfino Venuti in Rom gesandter Brief als Nachtrag hier erscheinen, den ich im Cod. Ottobon. des Vatikan 3128, Fol. 217, gefunden habe. Drei andere, die nach dem Index zur Ottoboniana an denselben Gelehrten gerichtet waren (Cod. Ottobon. 3126, Fol. 61, 99, 105), können leider nicht gegeben werden, weil der betreffende Band seit 1856 fehlt. $\mathrm{DaB}$ er gestohlen ist, wird als wenig wahrscheinlich bezeichnet 
(es war ein starkes, nicht leicht zu verbergendes Buch, das nur Briefe an Rud. Venuti enthielt), vielleicht ist er nur verstellt, so daß man immer noch auf ein Wiederfinden rechnen kann, vielleicht ist er aber auch durch die Feuchtigkeit ganz zerstört worden. Auf diesen Gedanken könnte man bald kommen, wenn man sieht, wie sehr auch die im Cod. Ottobon. 3128 enthaltenen Briefe einst gelitten haben.

Über $\mathrm{Ph}$. v. Stosch verweise ich auf den oben angeführten Beitrag. Rudolfino Venuti war 1705 in Cortona geboren; nach Beendigung seiner Studien ließ er sich zum Priester weihen und wurde nach längeren Reisen von Benedikt XIV. zum Präsidenten der Altertumskommission ernannt; er starb 1763. Seine Schriften über Altertümer sind zahlreich; über seine Wirksamkeit als Mitglied der Accademia di Cortona s. u. S. 333. Sein älterer Bruder Marcello, geb. 1700, studierte in Bologna und Siena und wurde frühzeitig von Don Carlos, dem nachherigen Könige beider Sizilien, mit der Verwaltung der Bibliothek und der Galeria Farnese, später auch mit der Aufsicht über die Herculanensischen Ausgrabungen betraut (er ist der erste, der mit der Description des premières découvertes faites dans l'antique cité d'Herculanum, Rome 1749, darüber Mitteilung gemacht hat). Dann kehrte er nach Cortona zurũck, wo er 1755 starb. Der dritte Bruder, Filippo Venuti, geb. 1709, wurde wie sein Bruder Rudolfino Priester; als solcher wurde er 1739 von dem Kapitel des Lateran nach Frankreich geschickt, um dort die in der Guyenne gelegenen Güter $z \mathfrak{u}$ verwalten; dort hat er großen Ruhm als Altertumsforscher erworben; 1750 kehrte er nach Rom und von da nach Cortona zurūck, wo er 1769 starb. An der Grūndung der Società Colombaria di Firenze, 1735, hat er neben Gori wesentlichen Anteil genommen.

$$
1 .
$$

Cod. Cortonese 497, fogl. 17.

a Florence le 24. fevrier 1739.

Monsieur.

Je me trouve honoré de votre lettre du 15. Janvier, que Votre frere Ridolfino m'a envoyée de Rome. Je lui envoie la presente pour Vous la faire parvenir avec l'incluse relation de 
l'Entrée joyeuse de L[eurs] A[ltesses] R[oyales] dans Florence. Votre medaille n'a pas ete executee. On grave le coin d'une autre inventee par l'Antiquaire Cochi ${ }^{1}$ ), ou le G[rand] $D$ [uc] est represente a cheval au quel Florence sortant de l'arc de Triomphe de Pière [1. pierre] (qui n'est pas ancor achevé a demi)") presente les clefs, avec l'inscription Adventus Principis felicissimi. Si jamais elle sera achevee, je tacherai de vous en procurer une de bronze. L'Inscription de l'arc des Juifs a ete fort approuvee. La maison de (raon ") a ete fort distinguee par le G. D. Mais il me parait que les Lorrains (voyant prattiquer a la cour une Etiquette fort differente de l'Antique de Lorraine familliaire) ") ne sont pas trop contents. Le G. D. n'admet personne a sa table a la reserve du seul Prince d'Elbœuf s). Les autres lui font la cour debout, quand il menge [l. mange] en Public. L'Enpereur a declaré le $\mathrm{G}$ [rand] prieur del Bene ${ }^{\circ}$ ) son conseiller Intime d'Etat avec le Titre d'Excellence, en lui accordant le rang sur Conte Herberstein i) Majorduomo de la G[rande] Duchesse. Les Florentins en

1) Antonio Cocchi, Philosoph und Naturforscher, von 1738-1758 Direktor des Museums der Uffizien. Vgl. A. Reumont, Gesch. Tosk. I, 332. K. Justi, Winckelmann, Il 2, 230.

2) Der zur Ankunft der neuen Herrscher errichtete Bogen auf dem Platze vor der Porta San Gallo, eine schwerfällige Nachahmung des Konstantinsbogens, ist erst später fertig geworden. Vgl. Reumont, Gesch. Tosk. II, 13.

3) Marc de Beauvau, Fürst von Craon, war als Beauftragter des Franz Stephan schon seit 1737 in Florenz eingetroffen, um die Übernahme des Thrones zu regeln. Vgl. A. Reumont, Gesch. Tosk. I, 499 u. a.

4) Die Etikette war am Florentiner Hofe ursprünglich nicht so steif wie an dem Kaiserlichen, wo das herkömmliche Zeremoniell des Reiches noch durch spanische Etikette gesteigert war. Vgl. Keyßler, Neueste Reisen I, 348. A. Reumont, Gesch. Tosk. I, 626; II, 7.

3) Es kann sich wohl nur um den Prinzen Eman. Maurice handeln (1677-1763), der 1706 in kaiserliche Dienste trat und gegen Ludwig XIV. kämpfte. Später kehrte er nach Frankreich zurück, wo er 1748. zum Herzog und Pair ernannt wurde.

6) Wahrscheinlich stammt er aus derselben florentinischen Familie wie Baccio del Bene, der in Diensten der Caterina de' Medici stand. Vgl. A. Reumont, Gesch. Tosk. I, 238.

7) Wohl ein Nachkomme des Frh. v. Herberstein, der von 1486 bis 1566 gelebt hat, dessen Bericht über RuBland (Rerum Moscoviticarum Commentarii) wiederholt gedruckt und vielfach übersetzt sind. 
General se louent beaucoup de l'Affabilité et des manieres familiaires et Gracieuses de L. A. R. Pendant le Carneval la cour a donne quatre grands bals dans le salone di Palazzo vecchio, ou toute[s] les masques ont ete admis. Deux fois s'est tenu le Gioco del Calcio $\left.{ }^{1}\right)$. Bernardin Riccardi Alfiere de la bande Rouge a gagne toute[s] les deux sur Folco Rinuccini Alfiere des Jeaunes. Le G. D. a donné a chaqûun des deux un diamant de deux cent Pistoles et des tabatieres d'or a ceux qui avait fait les plus beaux coups, et au Mannelli, ${ }^{2}$ ) qui avait gagne deux caccie, une boete [l. boite] guarnie de Diamants. Le dernier jour du Carneval devient malade d'un Rhume la G. duchesse, dont elle a eté fort incommode. Avant hier pour la premiere fois elle s'est promenee dans le jardin de Boboli. Enfin elle est guerie et enceinte de onze semaines. On dit communement, que toute[s] 3 L[eurs] A[ltesses] R[oyales] retourneront au mois de may a Vienne. ${ }^{3}$ ) Le G. D. n'en fait point de Mystere. Pour moi je suis de l'opinion, que le seul Prince Charles") fera un tour a Turin et de la se rendra tout droit a l'Armee en Hongrie.

1) Der Gioco del Calcio ist der heute wieder in Aufnahme gekommene FuBball, er wurde von zwei Parteien gespielt, deren Leiter den Namen Alfiere führten. Eine Unterabteilung des Gioco del Calcio waren die Caccie, Jagden, bei denen sich der junge Mannelli auszeichnete.

2) Wahrscheinlich der spätere Senatsprāsident, der im Jahre 1766 dem neuen Großherzog Leopold I. huldigte. A. Reumont, Gesch. Tosk. II, 78.

$\left.{ }^{3}\right)$ Schon nach drei Monaten verlieB Franz II. das Land, um nie wieder dorthin zurückzukehren. Vor seinem Scheiden hatte er die Regentschaft geordnet. Der Vorsitz wurde der verwitweten Kurfürstin von der Pfalz Maria Anna Louise, der Schwester des letzten GroBherzogs, die im Palazzo Pitti wohnte, angeboten, und als sie ablehnte, der Fürst von Craon zum Chef des Regentschaftsrates ernannt; ihm wurde der Graf von Richecourt für die Finanzen und Marchese Carlo Rinuccini für das Kriegswesen zugeordnet. Vgl. A. Reumont, Gesch. Tosk. II, 13.

4) Prinz Karl, der Bruder des GroBherzogs Franz Il., ist der als Feldherr aus dem Siebenjährigen Kriege bekannte Fürst. Über die Erziehung, die beiden Brūdern zuteil geworden, urteilt die Geschichte nicht so günstig, wie Phil. v. Stosch; vgl. A. Reumont, Gesch. Tosk. II, 7. Übrigens ist Prinz Karl nicht GroBherzog geworden, trotzdem er ursprünglich in Aussicht genommen war, für den Fall, daB Franz II. in 
Le prince montre beaucoup de gout pour les sciences et pour la mechanique, travaillant lui meme aussi bien a l'execution qu'a l'invention de machines de toute sorte. Selon toutes les Apparence[s] lui sera Granduc et aura pour femme la seconde Archiduchesse quand son Ayné sera fait Roy des Romains. L'un et l'autre de ces Princes ont une excellente education et le jugement propre pour Gouverner. J'aj ete presente a l'un et a l'autre et bongré malgre il a fallu comparaitre dorao ${ }^{1}$ ) comme les autres ce qui m'a coute septante Zeckins, c'est a dire en six mois je ne pourrai acheter ni medailles ni pieres gravees. ${ }^{2}$ ) Helás! Je ne deviendrai pourtant jamais bon courtisan, parceque l'air des Antichambres m'ennuit cruellement et les discours sur la plue [1. pluie] et beau temps assassaisonnes de quelque prise de tabac ancor davantage me deplaisent. Le Primat ${ }^{8}$ partit pour Rome pour se faire pretre le 7 . de Fevrier et y loge chez le devotissime Cardinal Bossu d'Alsace. ${ }^{4}$ ) Le Prince de Craon a ete fort maladiv cet hiver. Il me paroit que Passion d'animo est une des Principales causes. Je n'ay pas ancor assez parlé a Mr. Trussain ${ }^{5}$ ) pour pouvoir vous en faire le Caractere.

Österreich zur Regierung kommen sollte (vgl. Robiony, Gli ultimi dei Medici e la successione al Granducato di Toscana, Florenz 1905, S. 290), sondern Franz behielt, trotzdem er 1745 Kaiser wurde, die Herrschaft für sich, indem er sich durch eine Regentschaft vertreten lieB; nach seinem Tode 1765 wurde sein zweiter Sohn als Leopold I. Großherzog.

1) Dorao = dorato, d. h. in einem goldgestickten Hofgewande.

2) $\mathrm{DaB}$ es in bezug auf die Mittellosigkeit des Baron v. Stosch nicht so schlimm bestellt war, zeigen die weiter unten folgenden Mitteilungen über Ankäufe.

3) Um welchen Primas es sich handelt, läßt sich, wie es scheint, nicht bestimmen. DaB eine zum Primas ernannte Persönlichkeit erst nachher sich zum Priester weihen lieB, kommt öfter vor.

4) Vgl. Guarnacci, Vitae et res gestae Pontificum Romanorum et Cardinalium II, 329: Thomas Philippus de Alsatia Belga ex praeclarissimo Boussuviorum, nunc Principum Chimacensium sanguine ortus, 1679-1759. Berühmt sind die mannhaften Worte, die er an Ludwig XV. bei seinem Einzug in Brüssel richtete.

5) Das muB ein Vertrauter des Großherzogs sein, wahrscheinlich ein Lothringer. 
Je lui ay parlé une seule fois chez le Prince Craon. Il me paroit tres complaisant, ce que je reguarde pour une grande vertu a un ministre confident et favorit d'un grand souverain. Les comte Bernasconi de Milan et Fortunato Cervella di Ferrare examinent actuellement les offices delle Farine et fairont la meme chose a la Dogana, Magona, ${ }^{1}$ ) sale [1. sel] et autres branches des Revennues G. Ducales, qu'ils pretendent prendre en Ferme en avanceant au G. D. une somme d'un million et demi d'ecus florentins, lequel argent ils pretendent tirer des bourses Florentines sur l'esperance de grande usure. Je suis d'opinion, que cette Ferme rencontrera de difficultes Insurmontables, et si elle aura lieu non obstant cela, elle causera la ruine totale de cet Etat selon l'Idee, que je m'en suis formee des Finances de ce pais. ") Beaucoup des choses ont besoin de reforme, mais peu a peu, et pareille ferme ne pourra se mettre jamais en execution sans bouleverser entièrement tout le système du Gouvernement present. L. A. R. font de caresses extraordinaires à l'Electrice, ${ }^{3}$ ) qui en est charmee, et selon toutes les Apparences elle se chargera de la Regence avec Authorité au cas que la Cour retourne au de la des montes [1. montagnes]. Per legitima consequenza Rinuccini ${ }^{4}$ ) gagne journellement terrain et dans peu sera le despotique maitre. Ainsi Vous voyez bien que pour le present Il n'y a rien a esperer pour vous ${ }^{b}$ ) en Toscane et qu'il faut se contenter de Votre

1) Magona ist eigentlich eine Niederlage von Eisenwaren, wird in Toskana aber auch für jede Art von Magazin gesagt.

2) Über die traurigen Finanzverhältnisse Toskanas, die durch Verpfändung der Zölle nur noch verschlimmert wurden, vgl. A. Reumont, Gesch. Tosk. II, 26. Stosch hat sich hier als guten Propheten erwiesen.

3) Das ist die Kurfürstin Maria Anna Luise, Gattin des 1716 gestorbenen Kurfürsten Johann Wilhelm von der Pfalz, die Tochter des GroBherzogs Cosimo III. und Schwester des letzten Mediceerfürsten Johann Gasto. Sie war ursprünglich vom Senat als selbständige Herrscherin Toskanas vorgeschlagen worden.

4) Für Rinuccini vgl. oben S. 325, Anm. 3.

5) Filippo Venuti war damals in Clérac (Bourgogne), wohin er von dem Lateranensischen Kapitel geschickt war. $\mathrm{Er}$ muB wohl versucht haben, von dort wieder nach Italien zurückberufen zu werden; später (1747) wurde er zum Preposito di Livorno ernannt. 
Pathmos, jusquu a [ce] que ce Gouvernement a pris une certaine forme solide, pour scavoir a qui on doit s'adresser. Il $y$ a trois Jesuites confesseurs de L. A. R., loges dans le Palais Pitti. Hier Ils ont tenu dans leur Eglise magnifiquement paree Une Accademie en louange du G. Duc dont Lagomarsini ${ }^{1}$ ) a recité le Panegyrique, en quoj ils ont prevenu les autres gens de lettres, qu'on a deja commencé a depeindre a la Cour avec les couleurs les plus noires et odieuses en se servant des Impostures et mensonges a l'ordinaire de ces Messieurs. Eux sont toujours aux Oreilles des Princes, les gens de lettre, ordinairement peu Courtisans, restent presque toujours le sacrifice de la malignité et Imposture de leur adversaires. Enfin je Vous envie Votre charmante Retraite, ou vous pouves etudier a Votre aise sans songer aux Intrigues de la Cour. Ma Bibliotheque ${ }^{2}$ ) viendra surement a Livourne avant le mois de mais. Si nous etions alors ensemble, combien de belles choses pourrions nous expliquer. NB. les Jesuites deja ont fait courir le bruit que j'attendois ces balles de livres sous mon nom, touts livres defendus, pour les faire rendre a Rigacci, leur veritable proprietaire. A propos de Rigacci l'ordre de Rome est venu de lui rendre ses livres. Le Gouvernement tient en prison [secreta übergeschrieben] un certain Oloni qui avait assiste a l'Inquisiteur a faire la saisie des dits livres, et il ne sera relache si non apres la reddition de

1) Über Lagomarsini vgl. P. Tacchi-Venturi S. J., Corrispondenza inedita di L. A. Muratori con i padri Contucci, Lagomarsini e Orosz della Compagnia di Gesù, Roma 1901, S. 3.

2) Es ist nicht klar, ob die Bibliothek, die Stosch in Rom angelegt hat, so lange Zeit zurückgehalten worden ist, daB sie erst im Jahr 1739 in Livorno auf dem Seeweg anlangt, trotzdem Stosch schon 1731 Rom verlassen hat. Vielleicht handelt es sich um andere Bücher, die Stosch auf dem Seewege erwartet; dazu würde das in Florenz verbreitete Gerücht stimmen, der Eigentūmer der erwarteten Būcher sei eigentlich der Buchhändler Rigacci, der sich des Stosch nur bediene, um ungehindert von der Inquisition, die schon einmal sich seiner Būcherschätze bemächtigt hatte, sich neue Büchervorräte kommen zu lassen. Doch wahrscheinlicher muB man an die jedenfalls damals schon ziemlich bedeutende Bibliothek des Barons denken. 
touts les libres, desquels on voulait retenir Puffendorf et Vitriarius ${ }^{1}$ ) comme livres defendus. ") Enfin Cosino in genere di Cosini ha fatto la sua figura. ${ }^{3}$ ) Depuis Votre depart j'ai considerablement augmenté mes medailles de Villes Grecques. En les confrontant avec les gravees dans les livres, je trouve, que la pluspart sont tres mal dessinees et qu'il y a de quid pro quo Infinis, que Nos yeux, formes sur le[s] pieres gravees et marbres anciens, reconnoissent et que Golzius Paruta ${ }^{4}$ ) et autres n'ont pas compris. Celles qui sont dans le 1 . et troisieme Volume du Thesaurus Brandenburgicus de Laur. Begerus ${ }^{5}$ ), sont de toutes les plus correctement gravees et ressemblent le plus aux origineaux. Mais la vie humaine est trop courte. Vous et moy, que devions etre ensemble, sommes separés de cent lieues. A travailler seul on s'ennuit cruellement. Icy on ne peut se consulter avec personne, aussi le temps se passe en projets, que nos circonstances rendent inexecutables. $\mathrm{La}$ venue du Granduc et les depenses que la Noblesse a faites a cette occasion, ont causé la vente de bien de belles choses. J'ay achette quantité de excellentes figures Antiques

1) Samuel v. Pufendorf, 1632-1694; sein Hauptwerk, das hier wahrscheinlich gemeint ist, Traité du droit de la nature et des gens, hat viele Auflagen erlebt; ein früheres Werk De statu Imperii Germanici, Paris 1667, war in Wien auf den Index gesetzt worden. Nach längerer Tätigkeit in Schweden wurde er 1686 nach Berlin als Historiograph berufen. Mit Vitriarius ist wohl ein Werk des protest. Rechtslehrers Filippo Rinaldo Vitr. gemeint.

2) Der Versuch der Inquisition, die Veröffentlichung der Bücher zu überwachen, führte zu Zwistigkeiten zwischen dem Vatikan und dem Florentiner Hof, die erst durch das Einschreiten des Großherzogs beseitigt wurden; vgl. A. Reumont, Gesch. Tosk. II, 57.

3) Die Stelle ist wegen lokaler Beziehungen heute nicht recht verstăndlich. Es muß sich um einen kleinen, zu Intrigen geneigten Menschen handeln, der dem Stosch wie dem Venuti bekannt war.

4) H. Goltz, Icones, vitae et elogia Imperatorum Romanorum ex priscis numismatibus ad vivum delineatae, in Goltzii de re nummaria opera V. Antverpiae 1708. 2 vol. F. Paruta, La Sicilia descritta con medaglie. Palermo 1612. 2 vol.

s) L. Beger, Thesaurus Brandenburgicus selectus. Köln a. d. Spr. 1696-1700. 
de bronze. A la reserve du Lucumone di Culagna Il ny a point de Etruscherie ${ }^{1}$ ) par ici. Gori ${ }^{2}$ ) a bravement vendu a un certain B. Thoms? ses plus belles choses, entre autres sa statue de Marbre d'un Hiboux avec l'inscription Grecque en bas d'un Pronosticateur de choses a venir. ") Le Don Gel Patriarca aurait orné toute ma maison. ${ }^{8}$ ) Je ne m'etois jamais imaginé, que Gohri aurait vendu un monument si Remarquable, Auri sacra fames ${ }^{\circ}$ ) etc. Je n'ay rien voulu vendre au dit Thomas et j'ay refusé l'offre de sixcent sequins pour douze de mes anneaux. J'ay refusé le troc des diamants, dont il a été d'autant plus surpris, qu'il a trouve de Princes et Cardineaux plus traitables en Italie. Il a attrappé les plus belles pieres et Camees du monde. Il y a parmi quelquesuns avec des noms des graveurs, que j'ai fait dessiner, pour le [l. les] publier dans le supplement de

1) Über die Etruscheria vgl. K. Justi, Winckelmann II ${ }^{2}, 334$. Lucumone wurde der Präsident der Accademia Etrusca von Cortona genannt; die Anwendung des Ausdrucks hier hat scherzhafte lokale Bedeutung.

2) Franc. Gori, berühmter Altertumsforscher in Florenz, dessen Hauptwerk das Museum Etruscum ist, Florenz 1737-43.

3) Von diesem Thoms oder Thomas findet sich ein Bild bei P. L. Ghezzi, Cod. Ottobon. 3116, Fol. 19, verso. Er war 1737 in Rom, "huomo assai dilettante di Antichità e di Letteratura."

4) Wohin die Eule des Gori geraten ist, läßt sich bis jetzt nicht sagen. In der École des Beaux Arts zu Paris ist unter Nr. 3043 ein AbguB. Die Inschrift, bei der man das Wort $\mu a ́ v z \iota s$ erkennt, ist wohl modern.

5) Ph. v. Stosch hatte die Eule zu seinem Lieblingsvogel gemacht, so daB er immer die eine oder andere in seinem Zimmer hatte, wie er sagte, weil es ihn erheitere, ein Tier um sich zu sehen, das ihn selbst noch an Phlegma übertreffe. Auch auf den Bildern, die Ghezzi von Stosch gemalt hat (Cod. Ottobon. 3116, 1 und zum SchluB), ist immer eine Eule zugefügt; auf dem letzteren, dem großen Bilde der Antiquare, ist die Eule mit Nr. 1 als Don Gello bezeichnet, worin R. Kanzler (Un Congresso di Archeologi, Rom 1900, S. 8) merkwürdigerweise einen Antiquar verborgen glaubt. Die obige Stelle beweist, daB Don Gel einfach die im Stoschschen Hause übliche Bezeichnımg für die Eule war; vielleicht ist das Wort aus uccello verdorben. fames.

6) Verg. Aen. III, 56: quid non mortalia pectora cogis Auri sacra 
mon livre. ${ }^{1)}$ Je reconnais en plusieurs les debris du Gran Cabinet Farnesien ruiné par le fatal Jesuite et la barbarie des Espagnols et le nonchalence du Dernier Duc Antonio. ${ }^{2}$ ) Dont [l. donc], si j'etais a la place de Votre frere Marcelle ${ }^{3}$ ), je ne voudrais jamais etre Antiquaire si non sub beneficio legis et Inventarii. Je n'ay voulu achetter aucune des dites pieres, dont toute la Lombardie a été remplie, parce que je me souviens les avoir vues a Parme l'an 1714") et j'en avois tiré les cires de plusieurs et j'ay eu de Parme quantite de souffre[s], que autrefois avoit ete tiré [1. qui avaient été tirées] des Pieres du Cabinet Farnesien, qui me confirment dans l'opinion, que quantité de belles pieres du dit Cabinet ont ete depuis disperses et passé entre les mains de particuliers. Ce matin l'Amb. de Lugues a eu audience du G. D. pri [1. pris] a son autel [1. hôtel] par 2 mules de Cour. Il sera 3 jours traité aux dépens de la Cour au Casino de S. Marco. Je suis parfaitement, Monsieur, Votre tres humble et tres obeissant serviteur Philippe de Stosch.

Mon frere, $\left.{ }^{5}\right)$ Bonaccorsi, l'insigne Docteur Nagel et Marcus ${ }^{6}$ ) le peintre vous saluent.

1) $\mathrm{Zu}$ dem 1724 erschienenen Buche Phil. v. Stoschs Gemmae antiquae caelatae hatte der Verfasser einen Nachtrag geplant und viele Tafeln dazu schon stechen lassen. Doch ist der Plan nicht zur Ausführung gekommen. Die fertigen Kupferplatten scheinen spurlos verlorengegangen zu sein.

2) Der Herzog Antonio starb im Januar 1731. Über ihn vgl. KeyBler, Neueste Reisen Il, 1005. Die Reste des Farnesischen Kabinetts sind nach Neapel gekommen.

3) March. Marcello Venuti, der älteste der drei Brüder Venuti, war zum Vorsteher der Altertümer in Neapel ernannt und gab als solcher die Veranlassung $z u$ den Ausgrabungen in Herculanum. Später wurde er zugunsten des unfähigen Bajardi beiseite geschoben.

4) Stosch war damals zum ersten Male nach Italien gekommen.

5) Der Bruder des Philipp v. Stosch, Heinrich, starb in Florenz, vgl. Cod. Vat. Ottobon. 3116, Fol. 81. Nach Heinrichs Tode lieB Stosch seinen Neffen, den Sohn seiner an Prof. Muzel in Berlin verheirateten Schwester, kommen. Dieser hatte nach dem Tode des Philipp v. Stosch nichts Eiligeres zu tun als alle die gesammelten Schätze seines Oheims schleunigst zu Geld zu machen.

-) Marcus le peintre ist Marcus Tuscher, der mit den Brüdern Venuti durch enge Freundschaft verbunden war. $\mathrm{Er}$ arbeitete für Stosch. In Cortona, wo er zum Mitglied der Akademie ernannt war, ist er auch als Architekt tätig gewesen. Vgl. u. S. 335 f. 
Cod. Cort. 497, fol. 19.

Illmo. sigre sigre pre $\mathrm{Col}^{\mathrm{mo}}$.

Fire 11. Ottobre 1755.

Qesta mattina è comparso da me L'Abate Bartelemi ${ }^{1}$ ) e ha passato una mattinata a vedere le mie Medaglie Antiche, fra le quali ha trovato molte cose che mancano nel Tesoro regio di Francia. Io lo trovo il più dotto Antiquario che $\sin ^{\prime}$ ora ho conosciuto fra li Francesi, e quel che è molto raro a trovarsi di Là da Monti. Egli possiede quella parte della cognizione Antiquariesca, che si chiama pratica al supremo segno. Egli ha seco un Presidente del Parlamento di Parigi, gran dilettante di tutto quello, che spetta al disegno e all'Antichità. Il Conte di Caylus ${ }^{2}$ ) $\mathrm{mi}$ ha mandato un Esemplare magnificame legato del suo Libro Int! ${ }^{\circ}$ Recueil d'Antiquités Egyptiennes, Etrusques, Grecques et Romaines, con una Lettera molto obbligante nella quale il Sige Conte di Caylus in termini obbliganti mi domanda, che io gliene faccia la critica, è che gli comunichi il mio sentimento sopra diversi Monumenti da lui pubblicati, cosa che peraltro non ho intenzione di fare, perchè per quel poco che io sin ora ho potuto leggere, il libro stà benissimo. Come io adesso ne sono provvisto, non occorre, che V.S. Ill ${ }^{\mathrm{ma}}$ si pigli l'incomodo di farmi venir di Francia un Esemplare, ${ }^{3}$ ) ringrazziandola della benigna esibizione, che Ella mı fece l'ultima volta che io ebbi l'onore di

1) Jean Jacques Barthélemy, der bekannte Verfasser des Voyage du jeune Anacharsis en Grèce. $\operatorname{Er}$ war 1716 geboren und ist 1795 gestorben. Zum Direktor des Königlichen Münzkabinetts in Paris ernannt, machte er von 1754 bis 1757 eine Reise durch Italien, die er zu zahlreichen Erwerbungen für sein Kabinett benutzte.

2) Graf Caylus (Anne Claude Philippe de Tubières), 1692-1765, ist vielfach als Archäolog tătig gewesen. Sein Hauptwerk ist der oben erwähnte Recueil d'antiquités egyptiennes, étrusques, grecques, romaines et gaules, Paris $1752-67$.

3) Durch die in Clérac angeknūpften Verbindungen (Filippo Venuti war zum Mitglied der Akademie von Bordeaux u. a. ernannt) war er besonders 'geeignet, die in Frankreich erscheinenden Bücher zu besorgen 
vederla a Casa mia, in tal particolare, mi rassegno con tutto l'ossequio di V.S. Illma

$$
\text { devot }{ }^{\text {mo }} o b b^{\text {mo }} \text { Servre }
$$

Philippo de Stosch. ${ }^{1}$ )

All'Illmo Sigre Sigre e Pae Colmo

Mons $^{r}$ Venuti Proposto di Livorno.

3.

Cod. Ottobon. 3128, fol. 217.

Firenze 16. Agosto 1746.

\section{Monsieur.}

In replica alla sua Lettera de 13 . d. Le mando qui ingiunte 4 Stampe ragionevolmente male intagliate, che $\mathrm{L}^{\prime} \mathrm{Ab}^{\mathrm{e}} \mathrm{Va}$ lesio $\left.{ }^{2}\right)$ mi dette un giorno, senza dirmi à che appartenevano Li Originali, ed io L'avrei ignorato, Se L'Abe Sellari ') non mi avesse detto, che Le gemme appartenevano all'Accad ${ }^{2}$ Etrusca di Cortona. ') Le spiegazioni in margine sono del mentovato $\mathrm{Ab}^{\circ}$

1) Der Brief ist von einem Sekretär geschrieben und von Phil. v. Stosch nur unterschrieben.

2) Der Abbate Francesco Valesio, einer der berühmten Antiquare des XVIII. Jahrhunderts $(1670-1742)$, war dem Phil. v. Stosch durch enge Freundschaft verbunden, so daB er ihm den Text zu seinen Gemmae caelatae schrieb, unter der Bedingung, daB sein Name nicht genannt würde. Die Accademia di Cortona war am 29. Dezember 1726 von dem Abe Onofrio Baldelli in Rom mit seinen drei GroBneffen Marcello, Rudolfino und Filippo Venuti gegründet; sie sollte $\mathbf{4 0}$ gelehrte Kortonesen und 100 auswärtige Gelehrte zu Mitgliedern haben, zum Studium etruskischer Altertümer und zur Erläuterung neuentdeckter Denkmāler. Der Prāsident führte den Titel Lucumone; seit 1744 hielt die Akademie monatliche Versammlungen. Die Protokolle der Sitzungen wurden sorgsam aufbewahrt, eine Probe davon wurde 1751 von Gori veröffentlicht. AuBerdem lie $B$ die Akademie Dissertationen erscheinen, von 1736-1795 neun Bände, und im Jahre 1750 das Museum Cortonense, für das der Abbate Francesco Valesio Noten hinterlassen hatte, die von Gori und Rud. Venuti vermehrt wurden. Vgl. K. Justi, Winckelmann II $2,255$.

3) Ist vielleicht der Abe Gerolamo Sellari gemeint, der an der Kirche S. Maria in Via Lata Canonicus war und 1771 gestorben ist?

†) Vgl. Schreiber, Ber. d. sächs. Ges. d.W. 1885, S. 76. Die erwähnten Stampe waren wohl für das Museum Cortonense bestimmt (s. Anm. 2), sind aber dann wegen der hier mitgeteilten Bedenken nicht veröffentlicht 
Valesio, Le quali io suppongo egli abbia fatte estemporaneamente. La Stamp aSegnata Aa No. 4, spiegata Sorti Persiane, mi pare un pasticcio fatto à puro capr(iccio.) La credo moderna, e tale L'avrebbe creduta il Val(esio, se ne ave)sse veduta L'impronta. La stampa segnat( $a A)$ No. 2, battezzata Sagrifizio di M. Aurelio, è noto(riamente futta da un) Discepolo di Valerio Vicentino, ${ }^{1}$ ) e tale ('avrebbe pure giudica)ta il Valesio, se ne avesse veduta $\mathrm{L}^{\prime}$ (originale ........ Mess $\left({ }^{2}\right) \ldots$ c) hè sicuramente dise(gno . . . . . . . . . la morte $(\ldots \ldots$. . . . . )lzata [Rũckseite:]

pochi anni fà nel mio studio con altre Gemme antiche del med ${ }^{\circ}$; ed io ne portai a Roma il primo Solfo che è stato copiato da diversi moderni Artefici; onde ho creduto bene mandarle ne un'impronta fatta sul Originale, essendo intagliata in bellissima Corniola da tutte due Le parti, e V.S. III ${ }^{\text {ma }}$ Lo potrà confrontare colla gemma dell' Accad $^{2}$. Ho ancora presso di me diverse altre Stampe di Gemme antiche intagliate, che L'istesso $\mathrm{Ab}^{\mathrm{e}} \mathrm{Va}$ lesio mi regalò, che appartengono all'istessa opera, ma non essendovi niente scritto in margine giudico superfluo di mandargliele, supponendomi chè $\mathrm{Li}$ Tomi originali si trovino in mano dell'Ac$\mathrm{cad}^{2}$. - Gli Amici L'aspettano quà a Sett(embre, all)ora mi riserbo a farle vedere le mie raccolte. - In questo punto ricevo Lettera da un $\mathrm{A}\left(\right.$ mico $^{8}{ }^{8}$ ) che mi da La notizia che la Si(gnora .... di Roma) è venuta in questa città da .... Co(rtona?) [zweites Blatt (Fol. 218):]

quel Duomo; avendo Lasciato un peculio di circa m. 30 Scudi,

worden. - Der bekannte Karikaturenmaler Cav. Pier Leone Ghezzi hat das Bild des Valesio gezeichnet, Cod. Ottobon. 3114, f. 34, und die Bemerkung darunter gesetzt: ritratto dell'abbate Valesio morto il 22. Maggio $1742 \mathrm{di}$ 73 anni.

1) Belli, Valerio, gewöhnlich Valerio Vicentino genannt, wird als einer der gröBten Steinschneider bezeichnet. Er wurde 1479 in Vicenza oder Pesaro geboren und starb 1546; er arbeitete besonders für die Päpste Clemens VII. und Paul III., auch für Kardinal Bembo; ob einige in englischem Besitz vorhandene Steine ihm mit Recht zugeschrieben werden, muB fraglich bleiben. Vgl. Nagler's Künstlerlex. s. Belli.

2) Wohl ein Name.

ग) Hier fehlt der Name der Stadt. 
15 de quali li hà lasciato al do Sig. Ferreri, suo Sposo, e L'altra metà alla Sua Sorella di Genova; esso pure Sig. Ferreri che si diceva pericolosamente ammalato, dopo questa Eredità ha deposto ogni pensiere di malattia, ed ebbe tanta presenza di Spirito, di fare esso con il suo Cameriere L'Inventario della Robba della Defunta, nei tempo che se Le facevano Le Esequie in Duomo, e si allestiva di partirsene col suo bottino di ritorno in Patria. Addio Pietrelle, addio Medaglie, che seconddo Le app(arenze) non rivedranno piu Roma. Io non scrivo nien(te di q) uesto al Sig. Cardinale ${ }^{1}$ ), non avendomi mai (Sua Em)inenza (innanzi) fatta alcuna parola sopra il (tesoro di questa) Dama. Onde Se Lei lo vuole (communicare a) Baldani '), mi ferà piacere $\ldots \ldots$. . Le parole ....... Itre pa . . . . . . tra [Rückseite:]

quando avrò più tempo, e quando sarà meno caldo. Je suis parfaitement

P. S. Monsieur

Il Gatti se Le ricorda suo Umilo Servre.

Votre très humble et très obeissant serviteur Philippe de Stosch.

\section{Brief des Marcus Tuscher.}

In demselben Codex Ottobon. 3128, fol. 205, ist ein Brief des Marcus Tuscher enthalten, der jedenfalls auch an Venuti gerichtet war. Denn wenngleich die Adresse nicht erhalten ist, so spricht doch der Umstand, daß der Brief sich zwischen den Venutibriefen findet, für die Richtigkeit der Annahme. Marcus Tuscher, (vgl. Fr. Fr. Leitschuh in Beiträge zur Kunstgeschichte, Neue Folge 3, Verl. E. A. Seemann, Leipzig, S. 25) ist in Nürnberg 1705 geboren; an welchem Tage, war bis jetzt unbekannt; unser Brief zeigt, daB der 25. Mai sein Geburtstag war. Er wurde im Findelhause erzogen und zu dem Maler Joh. Dan. Preißler in die Lehre gegeben; 1728 ging er nach Rom, das er 1731 verlieB, um in

1) Jedenfalls ist Card. Alessandro Albani gemeint, zu dem Rud. Venuti in Beziehungen stand.

2) Das ist der Bibliothekar des Kardinals. 
das Haus des Baron v. Stosch in Florenz einzutreten. Durch Stosch ist er auch mit den bedeutendsten der rōmischen Antiquare in Verbindung gekommen (vergl. Naglers Künstlerlexikon s. v. Tuscher; dann schreibt man diesem Künstler auch zwei Medaillen zu, die eine auf seinen Gönner B. Ph. v. Stosch, die andere auf den Altertumsforscher Francesco Valesio), so daB auch die Bekanntschaft mit Rudolfino Venuti durchaus wahrscheinlich wird. 1734 ist Tuscher in Livorno, kehrte aber 1738 nach Florenz zurūck, das er 1741 verlieB, um nach London zu gehen, wo er die Herausgabe des ägyptischen Reisewerkes seines Freundes Norden übernahm; von dort wurde er nach Kopenhagen berufen als Hofmaler und Professor der Malerschule. Von dort stammt nun der Brief, den ich hier veröffentliche, vom 2. Mai 1747. Er hat sich des häuslichen Glückes, dessen Begründung er hier dem römischen Freunde mitteilt, nicht lange erfreuen können, denn schon im Jahre 1751 ist er gestorben. Über seine künstlerische Wirksamkeit und Bedeutung vgl. das oben angeführte Werk von Leitschuh, S. $48 \mathrm{ff}$.

\section{4.}

Cod. Ottobon. 3128, fol. 205.

Ill $^{\text {mo }}$ e Rev ${ }^{\text {mo }}$ Sigre Sige Pne mio Colmo!

L'Occasione ch'Ella si è degnata darmi, di poterla rinovare il mio Ossequio, mi è oltremodo gratissima, tanto più ch'avrei creduto ch'il pensare agli Iperborei Lidi avrebbe l'istesso Effetto del fiume Lete, a far dimenticarsi degli Amici dimoranti in questi Contorni, Ma l'esperienza per mia grandissima consolazione m'insegna omai del contrario; E S'Ella, mio Ottimo massimo Sig e Poe, dal Climate in cui presentemente respiro, avrà arguita che io mi sia dimenticato del mio Dovere in ver Persone a quali obligatissimo mi ricognosco, la presente divotissima mia Le persuaderà tutt' altrimente. Soggiornando in Italia ebbi Si grand' aversione contro questo Paese, che la sola Imaginazione d'un Baltico gelato, di Montagne tutt' il tempo estivo di Neve coperte, d'un Aere rigida, d'un suolo sterile e degli Abitatori, che mal leccati Orsi credetti, capace era di farmi passar la Voglia di trovarmi fra tante miserie umane, Ma nulladimeno 
eccomi quà, e che gran Gusto provo io, nel ved(ermi) disingannato d'un tal pregiudizio, trovando il l(ucido? mare) Baltico solcato da inumerabili concavi (gonfiati di vela), le Montagne amenissime, il Terreno (fertile, la città? in)fatti opposta per la Società civile.

[Rückseite:]

Le belle Arti e Scienze sono coltivate ed esercitate secondo il Grado dell' altezza polare, qui fassi in somma tutte le fonzioni corporee coll'istessa agilità e cerimonie che altrove. Ch'il bel sesso abbia il Suo Merito, V.S.Ill ${ }^{\mathrm{ma}}$ facilmente comprenderà da che Le so dire che alla fine ho stimato cosa conveniente, di liberarmi da una Libertà che in Sano Senso altro non è che una Impotenza di poter formare desiderj; e giacch'il Cor fissa Sempre da se medessimo il momento nel quale si dee rendere: $E$ che pertutto ove Si trova Beltà, s'adora come l'istessa Venere, Sendo la Bellezza si divina quant'Ella. In sostanza: Sono sposo con una Signorina che possiede quelle Virtù che rendono stimabile e desiderabile il suo sesso e atte sono a rendermi, mediante il Possesso di Lei, il più felice tra mortali, Secondo la mia ldea. Ella è figlia di questo regio Guardarobba della Galeria reale e Ritrattista della Corte, Uomo da bene, garbato e rispettabile, e Si chiama Giov. Salomone du Vaal. ${ }^{1}$ ) Il 25. di Maggio (come mio giorno natalizio) è il giorno appuntato per celebrare le Nozze; V.S.Illm ${ }^{m a}$ non può essere (presente, stando a) Roma, mà in Mente mia sarà e resterà (sempre amicissi)ma, e beveremo alla di Lei Conserva(zione ed al suo)

[2. Blatt:]

contento. Si degnerà dar parte di questo Evenimento à miei amici, che costà si trovano, e ciò facendo rispargneràaessi il Porto delle Lettere ed a me l'incommodo di Scriverle. Il $\mathbf{S}^{x}$

1) Joh. Salomon Wahl, vgl. Naglers Künstlerlex. XXI, 81 und Leitschuh a. a. o. S. 47: Er war geboren 1689 zu Chemnitz und frühzeitig in die Dienste des Königs von Dānemark gekommen; er machte sich durch eine große Anzahl von Bildnissen bekannt. J. M. PreiBler stach jenes des Königs Christian VI. und der Königin Juliana Maria. Diese Bilder, sowie solche von anderen Mitgliedern des kgl. Hauses, sind in der kgl. Gallerie und in den kgl. Schlössern usw. Wahl starb im Jahr 1763. 
Gramm ${ }^{1}$ ), Uomo erudito, Consigliere di Stato, Antiquario ed Archivario del Rè, non hà ancora voglia di benedire il temporale. Ma se in altrà qualità d'Antiquario, potrei aver'il piacere di vederla ben'accomodata qui, mel Sarebbe sommamente caro, carissimo. Le Novità di costà mi sono state riferite fedelmente dal $\mathrm{Sig}^{\mathrm{r}}$ Giorgio Naghel. ${ }^{2}$ ) Mi riverisca centupplicatamente la garbatissima $\mathrm{S}^{\mathrm{ra}}$ Francesca, La gentilissima Sigra Margherita ed altri amici ed amiche, conche rassegnandole la mia poco Valida Servitù, mi dò l'onore Soscrivermi inviolabilmente per sempre,

Di V.S.IIIma

Copenhaghen 2. Maggio 1747,

$$
\begin{array}{r}
\text { umil mo }^{\text {dev mo ed obligmo Servo Vero }} \\
\text { Marco Tuscher. }
\end{array}
$$

Si degni far vedere la qui acclusa

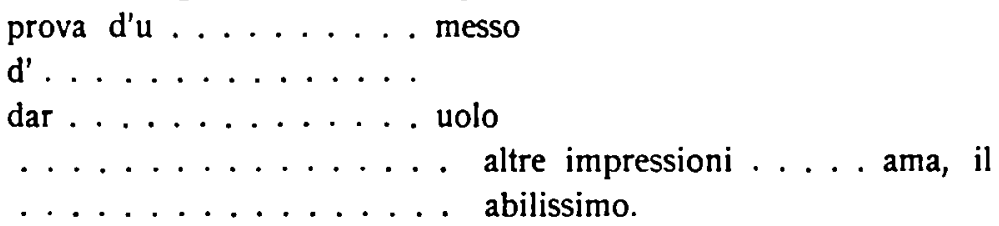

1) Joh. Gram war 1685 in einem Dorfe Jütlands geboren, wo sein Vater Pastor war. 1705 ging er nach Kopenhagen, um dort zu studieren, verōffentlichte 1706-1710 mehrere Dissertationen über philologische Themata, wurde 1714 zum Professor des Griechischen an der Universität und später zum Historiographen von Dänemark ernannt; er starb 1748. Er hat verhältnismäßig wenig veröffentlicht, hat aber den größten Einfluß3 innerhalb und außerhalb des Kōnigreiches ausgeübt. Vgl. Michaud, Biogr. univ. XVII, $317 \mathrm{ff}$.

2) Nagler, Künstlerlex. X, 102: Nagel, Georg Abraham, Zeichner und Maler, wurde 1712 zu Nürnberg geboren und von J. D. Preißler in der Kunst unterrichtet. Später ging er nach Italien, lebte sechs Jahre im Hause des Barons v. Stosch zu Florenz, zeichnete mehreres für diesen Kunstfreund, malte auBerdem auch Bildnisse und wurde $17+1$ sogar Mitglied der Florentinischen Akademie. Später ging er nach Kopenhagen, wo er drei Jahre blieb, bis er über Lübeck, Schwerin, Hamburg und Wien nach Rom sich begab, wo er um 1750 für den Kardinal Alexander Albanimehrere Bilder malte und seinen Ruf gründete. $\operatorname{Er}$ malte Bildnisse und Kirchenbilder. Starb 1779 in Rom. 